\title{
Perfil de Autoanticuerpos en Adultos con Trombocitopenia Inmune Primaria en una Institución de Bogotá.
}

\author{
Javier Mauricio Mora ${ }^{1}$, Alan Felipe Ovalle ${ }^{1}$, Carlos Augusto Solórzano ${ }^{1}$, Viviana Yiset López ${ }^{1}$, Pablo Cesar \\ Romero $^{2}$, Adriana Beltrán Ostos ${ }^{2}$
}

\begin{abstract}
${ }^{l}$ Departamento de Medicina Interna. Médico Internista, Hospital Universitario Clínica San Rafael. Bogotá, Colombia.
${ }^{2}$ Departamento de Medicina Intensiva y cuidado crítico. Hospital Universitario Clínica San Rafael. Bogotá, Colombia. ${ }^{2}$ Médico Internista Reumatólogo. Hospital Militar Central. Bogotá, Colombia.
\end{abstract}

\section{RESUMEN}

Introducción. Un recuento de plaquetas menor a 150,000 y normalidad en el resto del hemograma, son necesarios para considerar trombocitopenia inmune primaria (PTI). Aunque la patogénesis no ha sido enteramente dilucidada, se ha descrito una combinación de eventos caracterizados por aumento en la destrucción de las plaquetas mediados por la producción de autoanticuerpos. Publicaciones previas han reportado la presencia de autoanticuerpos en pacientes con PTI, anticuerpos anticardiolipina (75\%), de anticuerpos antinucleares (ANAS) $(33,3 \%)$ y anticuerpos antifosfolípido (AAF) $(28,5 \%)$. Estudios en nuestro país describen características demográficas y sociales de los pacientes con PTI así como la respuesta al tratamiento, pero no existen descripciones de la presencia de autoanticuerpos.

Objetivos. Identificar la prevalencia de autoanticuerpos en PTI, en el hospital universitario clínica San Rafael de Bogotá, en el periodo $2008-2012$.

Métodos. Se realizó un estudio observacional descriptivo, la población fue representada por adultos de ambos sexos mayores de 16 años con diagnóstico de trombocitopenia inmune primaria (PTI), en el hospital universitario clínica San Rafael de Bogotá, entre los años 2008-2012. Se excluyeron los pacientes con diagnostico confirmado de otra condición clínica como causa secundaria de trombocitopenia. Un total de 723 historias clínicas fueron revisadas, de las cuales 72 cumplieron los criterios de inclusión.

Resultados. De 72 historias clínicas analizadas, el 69,6\% correspondieron a mujeres, los autoanticuerpos positivos fueron los ANAS con 59,7\% seguido por los anticuerpos anticardiolipina (ACA) IgM con $18 \%$ y ACA IgG con $16.6 \%$, para el caso del anticoagulante lúpico se halló positivo en el $9,7 \%$. Los ANAS, positivos correspondieron a títulos $>$ a 1:160 y a su vez el patrón moteado fue el más frecuente para el $41,8 \%$, de los pacientes con recuento de plaquetas menores a 50,000 el 45\% tenían anticoagulante lúpico positivo. En el 30,5\% del total de casos se presentó alguna complicación, $59 \%$ fue sangrado.

Conclusiones. En este estudio hallamos que los anticuerpos más frecuentes fueron los ANAS y Anticuerpos Anticardiolipina.

Palabras clave: Trombocitopenia Inmune, Anticuerpos, ANAS, Anticoagulante Lúpico, Autoinmunidad.

\section{ABSTRACT}

Profile of Autoantibodies in Adults With Primary Inmune Thrombocytopenia in an Institution of Bogota.

Introduction. A platelet count below 150,000 and normality in the remainder of the blood count are necessary to consider primary immune thrombocytopenia (ITP). Although the pathogenesis has not been fully elucidated, a combination of events characterized by increased destruction of platelets mediated by autoantibody production has been described. Previous publications have reported the presence of Autoantibodies in patients with ITP, Anticardiolipin antibodies (AAC) (75\%), antinuclear antibodies (ANAS) (33,3\%) and Antiphospholipid antibodies (AAF) (28,5\%). Research in our country describe demographic, social characteristics and response to treatment of patients with ITP, but there are no descriptions of the presence of autoantibodies.

Objectives. To identify the prevalence of autoantibodies in PTI in the San Rafael clinical university hospital of Bogota, in the period $2008-2012$.

Methods. A descriptive observational study was carried out. The population was adults of both sexes older than 16 years old with diagnosis of Primary Immune Thrombocytopenia (ITP) at the San Rafael clinical university hospital of Bogotá between 2008-2012. Patients with another confirmed diagnosis as a secondary cause of thrombocytopenia were excluded. A total of 723 medical records were reviewed, of which 72 met the inclusion criteria.

Results. 72 medical records were analyzed, $69,6 \%$ were women, the positive autoantibodies were the ANAS with $59,7 \%$ followed by the antibodies Anticardiolipin (ACA) IgM with $18 \%$ and ACA IgG with $16,6 \%$, the lupus anticoagulant was positive in $9,7 \%$. The positive ANAS corresponded to titers $>1: 160$ and the mottled pattern was the most frequent with $41,8 \%$, of the patients with a platelet count lower than $50,000,45 \%$ had a positive lupus anticoagulant. In $30,5 \%$ of the total cases, some complication occurred, $59 \%$ were due to bleeding.

Conclusions. In this study we found that the most frequent antibodies were ANAS and Anticardiolipin Antibodies.

Keywords: Immune Thrombocytopenia, Autoantibodies, ANAS, lupus anticoagulant, Autoimmunity.

\section{*Autor de correspondencia: Carlos Augusto Solórzano catt21@hotmail.com}

Como citar: Mora, JM, Ovalle, AF, Solórzano, CA, López, VY, Romero, PC. Beltrán Ostos, A. Perfil de Autoanticuerpos en Adultos con Trombocitopenia Inmune Primaria en una Institución de Bogotá. Revista Cuarzo 2019;25(1):6-10.

Recibido: 23 de noviembre de 2018 Aceptado: 5 de febrero de 2019 Publicado: 30 de junio de 2019

DOI:https://doi.org/10.26752/cuarzo.v25.n1.346 


\section{INTRODUCCIÓN}

$\mathrm{L}$ a trombocitopenia inmune primaria (PTI), es una enfermedad autoinmune adquirida, que se caracteriza por la presencia de un recuento de plaquetas en sangre periférica $<150,000 / \mathrm{L}$ (1) con normalidad en el resto del hemograma, otros autores establecen como valor de referencia un recuento menor a $100.000 / \mathrm{L}$ dado que $6,9 \%$ entre $100,000 \mathrm{y}$ 150,000 presentan trombocitopenias transitorias (2). Los estudios de prevalencia mejor caracterizados en relación a PTI, se han realizado en Estados Unidos, dos ejemplos de esto son el estudio de Segal y Powe, quienes estimaron que la prevalencia fue de 9,5 casos por 100,000 habitantes, en hombres de 6.1 y en mujeres 11,3 por 100,000 habitantes respectivamente; por otro lado, Feudjo-Tepie et al., describieron prevalencia en PTI crónica en mayores de 65 años de 38,3 por 100,000 habitantes $(3,4)$.

Debe tenerse en cuenta que el diagnóstico de la enfermedad se establece luego de descartar otras causas de trombocitopenia. Las manifestaciones clínicas de la enfermedad son variables, $1 / 3$ de los pacientes no presenta sintomatología, quienes la presentan está dada por petequias de predominio en extremidades inferiores, región sacra y sangrados que van desde cuadros leves como la epistaxis hasta hemorragias que ponen en riesgo la vida, entre ellas la hemorragia intracerebral y la hemorragia gastrointestinal las cuales ocurren en menos de $1 \%$ de los casos (2-5).

Aunque la patogénesis de la PTI no ha sido enteramente dilucidada, se ha descrito una combinación de eventos caracterizados por aumento en la destrucción de las plaquetas mediadas por la producción de autoanticuerpos, asociado a la inhibición de la producción de plaquetas (6).

Los autoanticuerpos juegan un papel preponderante en la patogénesis que resulta en el mecanismo final que activa la destrucción plaquetaria, sin embargo, previo a esto, hay tanto mecanismos iniciadores como perpetuadores involucrando a células presentadoras de antígenos, linfocitos B y T (7). Estas células reconocen por algún motivo no claro a autoantígenos plaquetarios como las glicoproteínas (GP) IIb-IIIa y Ib-IX; Kuwana et al., (1998) encontró linfocitos B y T autoreactivos a fragmentos de GP no propios (7).

La pérdida de tolerancia postimica como periférica facilita la supervivencia de clonas de linfocitos B y $\mathrm{T}$ autoreactivos, posteriormente se activan señales coestimuladoras CD 40CD40 o CD 28- CD28 durante la exposición a otros antígenos externos (virus, bacterias) que incrementan el número de autoanticuerpos; también podrían estar relacionadas moléculas del complejo mayor de histocompatibilidad clase 1 incrementando la expresión de CD 40 (6). Por otro lado, el incremento de niveles de factor de crecimiento transformante beta 1 mediada por linfocitos $\mathrm{T}$ inhibe la maduración de megacariocito a plaqueta en la medula ósea (8).

Se ha venido ampliando el listado de autoanticuerpos presentes en PTI, en un estudio prospectivo realizado en Corea del Sur entre enero de 2003 y diciembre de 2008 en donde se reclutaron 60 pacientes con edad media de 48 años, se encontró que 20 pacientes tenían anticuerpos antifosfolípido (AAF) $(28,5 \%)$, de los cuales $15(75 \%)$ tenían solo anticuerpos anticardiolipina (AAC), 2 (10\%) tenían anticuerpos anticardiolipina (ACA) y anticoagulante lúpico (ACL), y 3 (15\%) solo tenían anticoagulante lúpico(9). Reyhand et al., en una muestra de 82 pacientes con PTI recién diagnosticados encontraron la presencia de ACL, ACA IgG e IgM y AAF, 31 pacientes $(37,8 \%)$ tenían anticuerpos antifosfolípidos positivos al momento del diagnóstico sin encontrar diferencias significativas respecto al conteo de plaquetas y respuesta al tratamiento; sin embargo a los 5 años de seguimiento el grupo de antifosfolípidos positivos y negativos mostraron supervivencia acumulada libre de trombosis de 39\% y 97,7\%, respectivamente (10). Por su parte Stasi et al., encontraron anticuerpos antifosfolípidos presentes en $40 \%$ de pacientes con PTI (2).

Abdullah et al., hallaron que un 33,3\% de adultos con PTI tienen títulos de ANAS positivos $>1: 80$, sin embargo ninguno en el seguimiento a 3,5 años desarrollo lupus eritematosos sistémico (LES) (11), al parecer esto es un predictor de evolución a PTI crónica en niños sin haber evidencia de esto en adultos; del 8 al 14\% de positividad de anticuerpos antitiroideos se asoció con trombocitopenia moderada y desarrollo de enfermedad tiroidea autoinmune, la función tiroidea normal coincidió con resolución PTI (12).

En Colombia existen estudios descriptivos como los de L. A Ramírez en Armenia y J. Aduen en Bogotá, en los que se describen características demográficas y sociales de los pacientes con PTI así como la respuesta al tratamiento, sin embargo en dichos artículos no se describe la presencia de autoanticuerpos $(13,14)$.

El propósito de nuestro estudio es describir el perfil de auto anticuerpos en pacientes con trombocitopenia inmune primaria, en el Hospital Universitario Clínica San Rafael de Bogotá, en el periodo $2008-2012$.

\section{MATERIALES Y MÉTODOS}

Se realizó un estudio observacional descriptivo, con base en registros clínicos de pacientes adultos de ambos sexos mayores de 16 años con diagnóstico de trombocitopenia inmune primaria (PTI); en el hospital universitario clínica San Rafael de Bogotá, que consultaron entre los años 2008-2012.

Se incluyeron registros clínicos de pacientes con diagnóstico de PTI definido como un recuento plaquetario $<150,000 / \mathrm{L}$ a los cuales por protocolo institucional se les solicitó perfil inmunológico (anticuerpos antinucleares, anticuerpos extractables de neutrófilo, complemento, AAF, AAC, ACL) y fueron considerados para inclusión aquellos con al menos un autoanticuerpo positivo. Se definieron como criterios de exclusión pacientes con diagnostico confirmado de VIH, Hepatitis B y C, cáncer, artritis reumatoide, lupus eritematosos 
sistémico, síndrome antifosfolípido, vasculitis como causa secundaria de trombocitopenia.

No se realizó cálculo del tamaño de la muestra, dado que fueron analizadas todas las historias clínicas obtenidas.

Las variables a estudio fueron: Género, ANAS, patrón de ANAS, anti Sm, anti RNP, anti $\mathrm{La}$, anti Ro, anticoagulante lúpico, anti DNA, complemento $\mathrm{C} 3$, complemento $\mathrm{C} 4$, anti DNA, necesidad de uso de corticoide, respuesta a corticoide, antecedentes familiares de enfermedad autoinmune.

Se caracterizó por sexo y edad a la población en estudio, se calculó la frecuencia de los autoanticuerpos, con sus correspondientes intervalos de confianza del 95\% (IC 95\%). Se calcularon medidas de tendencia central y dispersión. Se consideraron significativas las diferencias con un valor de $\mathrm{p}<$ 0,05 . Para realizar el análisis estadístico se utilizó el programa SPSS versión 22.0 de IBM y Microsoft Excel -2010.

En cuanto a los aspectos éticos, esta investigación se clasifica como sin riesgo, sustentado en la resolución 8430 de 1993 del ministerio de salud (hoy de salud y protección social), la cual es una investigación documental que no interviene o modifica las variables biológicas o clínicas de los pacientes que ingresaron al hospital y participaron en el estudio; manteniéndose la confidencialidad de la información obtenida de las historias clínicas según la resolución 1995 de 1999. Hubo confidencialidad absoluta de todos los datos. Estos fueron guardados en tres computadores registrados, y que son parte de los investigadores.

\section{RESULTADOS}

Se revisaron un total de 723 historias clínicas, de las cuales 72 cumplieron con los criterios de inclusión, en las que el 69,6\% de la población a estudio correspondió a mujeres. El promedio de edad de la población general fue de 45,2 años desviación estándar (DE) 18,5, la edad mínima fue de 16 años y la edad máxima de 88 años, para los mayores de 65 años la frecuencia fue del $22 \%$. El $44 \%$ del total de pacientes presentaron valores de plaquetas $<50,000 / \mathrm{L}$.

Se encontró que los anticuerpos más frecuentes fueron los ANAS en 43 casos $(59,7 \%)$, seguido por los anticuerpos anticardiolipina (ACA) IgM con 13 casos (18\%) y ACA IgG con 12 casos $(16,6 \%)$, para el caso del (ACL) se halló positivo en 7 casos que representa el 9,7\% del total (tabla 1). Los ANAS, positivos correspondieron a títulos $>1: 160$ en el $39,5 \%$, y a su vez el patrón moteado fue el más frecuente con 18 casos $(41,8 \%)$.

Adicionalmente se encontró que de los pacientes con recuento de plaquetas menor a 50,000, el 45\% (18) tenían anticoagulante lúpico positivo, el $17.5 \%$ (7) tenían anticuerpos anticardiolipinas (ACA) IgM positivo y otro $17,5 \%$ (7) tenían ACA IgG igualmente positivo (Tabla 1).

En relación con las complicaciones, el 30,5\% (22) de los 72 casos presentaron algún tipo de complicación, en donde el sangrado fue el más frecuentemente registrada representando el $59 \%$ (13) seguido por los eventos trombóticos venosos con el $41 \%$ (9) casos, no se registró ningún caso de trombosis arterial (Tabla 2).

Tabla 1: Distribución de Anticuerpos.

\begin{tabular}{|c|c|c|c|}
\hline Anticuerpos & Numero & Porcentaje & IC $95 \%$ \\
\hline$A N A S$ & 43 & 59,7 & $47,4-71,1$ \\
\hline$A C A \operatorname{Ig} M$ & 13 & 18,1 & $9,9-28,8$ \\
\hline$A C A \operatorname{Ig} G$ & 12 & 16,7 & $8,9-27,3$ \\
\hline$A C L$ & 7 & 9,7 & $3,9-19$ \\
\hline$A N T I D N A$ & 5 & 6,9 & $2,2-15,4$ \\
\hline$A F L \operatorname{Ig} M$ & 2 & 2,8 & $0,3-9,6$ \\
\hline$A N T I R N P$ & 2 & 2,8 & $0,3-9,6$ \\
\hline$A F L \operatorname{Ig} G$ & 1 & 1,4 & $0,003-0,074$ \\
\hline B $2 \operatorname{Ig} G$ & 1 & 1,4 & $0,003-0,074$ \\
\hline B $2 \operatorname{Ig} M$ & 0 & 0 & 0 \\
\hline Ac $S M$ & 0 & 0 & 0 \\
\hline ANTI RO & 0 & 0 & 0 \\
\hline$A N T I L A$ & 0 & 0 & 0 \\
\hline
\end{tabular}

Listado de autoanticuerpos encontrados, discriminando frecuencia absoluta, porcentajes e intervalos de confianza en columnas de izquierda a derecha respectivamente.

Tabla 2: Proporción de Anticuerpos Según Complicaciones.

\begin{tabular}{|c|c|c|c|c|c|c|}
\hline \multicolumn{4}{|c|}{ Trombosis venosa (n 9) } & \multicolumn{3}{|c|}{ Sangrado (n 13) } \\
\hline Anticuerpos & \# & $\%$ & IC $95 \%$ & \# & $\%$ & IC $95 \%$ \\
\hline$A N A S$ & 4 & 5,6 & $1,5-13,6$ & 9 & 12,5 & $5,8-22,4$ \\
\hline$A C A \operatorname{Ig} M$ & 3 & 4,2 & $0,8-11,6$ & 1 & 1,4 & $0,003-0,074$ \\
\hline$A C A \operatorname{IgG}$ & 3 & 4,2 & $0,8-11,6$ & 1 & 1,4 & $0,003-0,074$ \\
\hline$A C L$ & 3 & 4,2 & $0,8-11,6$ & 1 & 1,4 & $0,003-0,074$ \\
\hline$A N T I D N A$ & 1 & 1,4 & $0,003-0,074$ & 0 & 0 & 0 \\
\hline$A F L \operatorname{Ig} M$ & 1 & 1,4 & $0,003-0,074$ & 0 & 0 & 0 \\
\hline ANTI RNP & 1 & 1,4 & $0,003-0,074$ & 0 & 0 & 0 \\
\hline$A F L \operatorname{Ig} G$ & 0 & 0 & 0 & 0 & 0 & 0 \\
\hline B $2 \operatorname{Ig} G$ & 0 & 0 & 0 & 0 & 0 & 0 \\
\hline B $2 \operatorname{Ig} M$ & 0 & 0 & 0 & 0 & 0 & 0 \\
\hline$A N T I S M$ & 0 & 0 & 0 & 0 & 0 & 0 \\
\hline$A N T I R O$ & 0 & 0 & 0 & 0 & 0 & 0 \\
\hline$A N T I L A$ & 0 & 0 & 0 & 0 & 0 & 0 \\
\hline
\end{tabular}

frecuencias.

Dentro de los tratamientos suministrados, el más usado fue corticoide en el 93\% (67) de los casos, de los cuales el 79,1\% (53) tuvo respuesta completa, definida como recuentos plaquetarios $>100,000 / \mathrm{L}$ después de 3 meses de tratamiento.

Tomando las variables grado de trombocitopenia en dos estratos mayor y menor a 100,000 plaquetas y ANAS, se encontró distribución normal por lo que se realizó prueba de correlación de Pearson obteniendo un valor de p 0,684 no hubo significancia en la correlación en este grupo de paciente. Adicionalmente se calculó la medida de asociación (OR 0,88 IC 95\% 0,23:3,42) encontrando disminución del riesgo de trombocitopenia menor a 100,000 en aquellos pacientes con ANAS negativos sin embargo esta asociación no es estadísticamente significativa. 
Para el caso de las variables anticuerpos anticardiolipina y trombosis venosa se comportaron también de manera normal por lo que se realizó la prueba de correlación paramétrica sin significación estadística $(\mathrm{p}=0,38)$, para el caso de las pruebas de asociación se encontró que tener anticuerpos anticardiolipina positivos aumentaba en 2,6 veces el riesgo de desarrollar trombosis venosa, pero sin diferencias estadísticamente significativas (OR 2,6 IC 95\% 0,35-19,1).

\section{DISCUSIÓN}

En nuestro estudio los autoanticuerpos en pacientes con trombocitopenia inmune primaria se presentaron en ambos sexos, con un claro predominio para el sexo femenino, que concuerda con los hallazgos de Segal y Powe. La distribución por edades según un estudio publicado por Feudjo et al., con 4943 pacientes en Estados Unidos reporta frecuencia de 18\% en mayores de 65 años de edad versus el $22 \%$ para el mismo grupo de edad documentado en nuestro estudio.

Respecto al perfil de autoanticuerpos, no se encontró ninguna publicación que evaluara la frecuencia de la totalidad de los autoanticuerpos incluidos en este estudio, sin embargo para el caso de los ANAS se hallaron positivos en el 59,7\% de los pacientes, que difiere de lo encontrado por Abdullah et al., cuya frecuencia fue del $33 \%$, pero se debe aclarar que en dicho estudio se aceptaron títulos desde 1:80 lo que explicaría la diferencia ya que en nuestro caso se incluyeron como positivos títulos mayores a 1:160.

Otros autoanticuerpos que se destacan son los ACA tanto IgM como IgG, que en estudios previos en los que se evaluó su frecuencia, 26,6\% según datos de Yang et al., en 2011 con 70 pacientes, difiere de nuestros resultados en donde fueron positivos en el 34,6\%, aunque el tamaño de muestra es equiparable (70 vs 72) hay diferencias en los criterios de inclusión puntualmente para el conteo plaquetario el cual fue menor de 100,000 y menor de 150,000 respectivamente. Para el caso del anticoagulante lúpico que fue positivo en el 9,7\% de nuestros pacientes, es menor a lo reportado (24,3\%) en un estudio turco con 82 participantes $(9,10,11)$, el cual revela también que el $45 \%$ de los participantes tuvieron trombocitopenia severa, con complicaciones como sangrado y trombosis venosa se encontró frecuencias de autoanticuerpos semejantes a nuestros datos. Del total de los casos incluidos se registró un 12,5\% de trombosis venosa, sin hallar eventos trombóticos arteriales. En una publicación en el seguimiento a 5 años se encontró presencia de enfermedad trombótica en pacientes con AAF, ACA y ACL (10). Los ANAS no tiene antecedente de publicación con relación a eventos trombóticos, sin embargo 4 de nuestros pacientes presentaron trombosis venosa, aun así, se ha descrito progresión de la trombocitopenia en los pacientes con ANAS positivos (11).

Por otro lado, el sangrado fue la complicación más frecuentemente hallada en nuestro estudio con 59\% (13) de los casos, relacionados con ANAS (9 pacientes) condición sin antecedentes en otras publicaciones, seguido de ACA IgG, ACA IgM y ACL con un caso cada uno. Se debe tener en cuenta que títulos positivos de ANAS se pueden encontrar en la población general hasta en un 9,5\% (11), o a su vez se pueden encontrar títulos positivos de ANAS entre 8-10 años previo al diagnóstico de LES cómo se ha registrado en otras publicaciones $(15,16)$.

En este trabajo no se midieron los anticuerpos antitiroideos los cuales han sido descritos en publicaciones relacionadas al tema, por lo anterior sugerimos sean incluidos en futuros protocolos de investigación. Es probable que futuros estudios de tipo prospectivo nos aporte información sobre la relación entre anticuerpos y riesgo de trombosis o de sangrado, más aún en el escenario de los pacientes con ANAS positivos de los cuales no se cuenta con antecedentes registrados en la literatura, de igual manera evaluar si un grado leve de trombocitopenia podría comportarse como un factor protector para el desarrollo de complicaciones.

Conflicto de Interés: Los autores no declaran conflicto de interés.

\section{REFERENCIAS}

1. Rodeghiero F, Stasi R, Gernsheimer T, et al. Standardization of terminology, definitions and outcome criteria in immune thrombocytopenic purpura of adults and children: report from an international working group. Blood. 2009;113(11): 2386-2393.

2. Stasi R, Amadori S, Osborn J, Newland AC, Provan D. Long-term outcome of otherwise healthy individuals with incidentally discovered borderline thrombocytopenia. PLoS Med. 2006; 3(3):e24.

3. Segal, J. B.; Powe, N. R. Prevalence of immune thrombocytopenia: analyses of administrative data. Journal of Thrombosis and Haemostasis, 2006, vol. 4, no 11, p. 2377-2383.

4. Feudjo-Tepie, M. A., N. J. Robinson, and D. Bennett. "Prevalence of diagnosed chronic immune.

5. Moulis G, Palmaro A, Montastruc JL, et al. Epidemiology of incident immune thrombocytopenia: a nationwide populationbased study in France. Blood 2014;124(22):3308.

6. Cooper N, Bussel J. La patogénesis de la púrpura trombocitopénica inmune. Br J Haematol 2006; 133: 364.

7. Chong BH: Primary immune thrombocitopenia: understanding pathogenesis is the key to better treatments. J Thromb Haemost 2009; 7:319.

8. Yoshimura, C., Nomura, S., Nagahama, M., Ozaki, Y., Kagawa, H. \& Fukuhara, S. (2000) Plasma-soluble Fas (APO-1, CD95) and soluble Fas ligand in immune thrombocytopenic purpura. European Journal of Haematology, 64, 219-224.

9. Yang YJ, Yun GW, Song IC, Baek SW, Lee KS, Ryu HW, Lee MW, Lee HJ, Yun HJ, Kim S, Jo DY. Clinical implications of elevated antiphospholipid antibodies in adult patients with primary immune thrombocytopenia. Korean J Intern Med. 2011 Dec; 26(4):449-54.

10. Reyhan Diz-Kucukkaya, Abdullah Hacihanefioglu. Antiphospholipid antibodies and antiphospholipid syndrome in patients presenting with immune thrombocytopenic purpura: a prospective cohort study. Blood Journal 2001;98(6):1760-1764.

11. Alintas A; Prevalence and clinical significance of elevated antinuclear antibody test in children and adult patients with idiopathic thrombocytopenic purpura; J Thromb Thrombolysis; 2007 Oct;24(2):163-8. Epub 2007 Apr 14. 
12. Provan D, Stasi R, Newland AC, et al. International consensus report on the investigation and management of primary immune thrombocytopenia. Blood 2010;115:168-186.

13. L. A. Ramírez, M.P. cabrera, N.L. Arredondo. Purpura trombocitopenica autoinmune: experiencia de cinco años en el hospital universitario san juan de dios de armenia. acta médica colombiana vol. 13 no. 5 (septiembre-octubre) 1988.

14. Arduen $\mathrm{j}$, et al. Púrpura trombocitopénica idiopática en pacientes tratados en el hospital san juan de dios, Bogotá (1980-1985). Salud uninorte 4-5 (1): 19-26.

15. Kurata Y; High-titer antinuclear antibodies, anti-SSA/Ro antibodies and anti-nuclear RNP antibodies in patients with idiopathic thrombocytopenic purpura.Thromb Hemost; 1994 Feb;71(2):184-7.

16. Katja M. Clinical and laboratory predictors of chronic immune thrombocytopenia in children: a systematic review and metaanalysis BLOOD, 20 November 2014 x volume 124, number 22. 